\title{
Mycorhization contrôlée et fertilisation phosphatée : applications à la domestication du jujubier
}

Amadou Bâa $^{\star}$, Tiby Guissou ${ }^{b}$, Robin Duponnois ${ }^{c}$, Christian Plenchette $^{d}$, Ousmane Sacko ${ }^{e}$, Daouda Sidibéf, Kondé Syllag, Baba Windoug

\author{
a Laboratoire de Microbiologie \\ des sols, Centre de recherche \\ IRD / Isra, BP 1386, \\ Dakar, Sénégal \\ b Laboratoire de Microbiologie \\ forestière, \\ DPF / Inera / CNRST, \\ BP 7047 , \\ Ouagadougou 03, \\ Burkina Faso
}

c Laboratoire de Bio-pédologie, Centre de recherche IRD / Isra, BP 1386, Dakar, Sénégal

d Station d'Agronomie, Inra, BP 86510, 17 rue Sully, 21065 Dijon, France

e École normale supérieure, BP 241, Bamako, Mali

f Institut d’Économie rurale, BP 270, Bamako, Mali

9 Direction des Eaux et forêts, chasse et de la conservation des sols et Projet de Reboisement villageois dans le nord-ouest du Bassin arachidier, BP 338 A, Thiès, Sénégal

amadou.ba@univ-ag.fr

* Correspondance et tirés à part

Reçu le 13 juillet 2000 Accepté le 12 mars 2001

Fruits, 2001, vol. 56, p. 261-269 (c) 2001 Cirad/EDP Sciences All rights reserved

RESUMEN ESPAÑoL, p. 269

\section{Controlled mycorrhizal infection and phosphate fertilizer use:} applications to the domestication of the jujube tree.

Abstract - Introduction. The jujube tree, Zizyphus mauritiana Lam., is a forest fruit tree required for its fruit by the populations of the Sahel. The species domestication was approached starting from silvicultural techniques like controlled mycorrhizal infection and fertilization with rock phosphates (RP). This document presents a summary of the results acquired on these subjects. Materials and methods. The mycorrhizal dependence of the jujube tree and of twelve other forest fruit tree species was studied regarding two arbuscular mycorrhizal fungi, Glomus aggregatum and G. intraradices. In addition, the capacity of the tree for mobilizing phosphorus from West Africa RP was evaluated in sandy soil, low in assimilable phosphorus and to a slightly acid pH. Jujube trees inoculated with $\mathrm{G}$. aggregatum were then put in the field to follow growth parameters. Results and discussion. Among the thirteen forest fruit tree species studied, the jujube tree proved to be most dependent on mycorrhizal symbiosis with G. aggregatum which acts on its growth and its supply with assimilable phosphorus. The mycorrhized jujube trees mobilize more effectively the phosphorus of the Tilemsi (Mali) RP that those of the Taiba (Senegal) and Kodjari (Burkina Faso) RP. In tree nurseries, controlled mycorrhizal infection has beneficial effects on the growth of jujube trees of various origins. In plantation, this beneficial effect of the inoculation was also observed on some 20 month old trees. Conclusion. The techniques of controlled mycorrhizal infection and fertilization with $\mathrm{RP}$ are ready to improve the growth and the phosphatic nutrition of the jujube trees in tree nurseries and the field. However, they will have to be tested in other forest sites to evaluate their effects on the fodder and fruit-bearing production of the jujube trees.

West africa / Zizyphus mauritiana / domestication / mycorrhizal infection / arbuscular mycorrhizae / fertilizer application / rock phosphate / phosphorus

\section{Mycorhization contrôlée et fertilisation phosphatée :} applications à la domestication du jujubier.

Résumé - Introduction. Le jujubier, Zizyphus mauritiana Lam., est un arbre fruitier forestier très recherché pour ses fruits par les populations du Sahel. La domestication de cette espèce a été abordée à partir de techniques sylvicoles comme la mycorhization contrôlée et la fertilisation phosphatée avec des phosphates naturels (PN). Le document présente une synthèse des résultats acquis sur ces sujets. Matériel et méthodes. La dépendance mycorhizienne du jujubier et de douze autres espèces d'arbres fruitiers forestiers vis-à-vis de deux champignons mycorhiziens à arbuscules Glomus aggregatum et G. intraradices a été étudiée. Par ailleurs, la capacité de l'arbre à mobiliser du phosphore à partir de PN d'Afrique de l'Ouest a été évaluée dans un sol sableux, pauvre en phosphore assimilable et à $\mathrm{pH}$ faiblement acide. Des jujubiers inoculés avec $\mathrm{G}$. aggregatum ont été ensuite mis au champ afin de suivre des paramètres de croissance. Résultats et discussion. Parmi les treize espèces d'arbres fruitiers forestiers étudiés, le jujubier s'est révélé être le plus dépendant de la symbiose mycorhizienne avec G. aggregatum qui agit sur sa croissance et son alimentation en phosphore assimilable. Les jujubiers mycorhizés mobilisent plus efficacement le phosphore des PN de Tilemsi (Mali) que celui des PN de Taïba (Sénégal) et de Kodjari (Burkina Faso). En pépinières, la mycorhization contrôlée a des effets bénéfiques sur la croissance de jujubiers de différentes provenances. Cet effet bénéfique de l'inoculation a aussi été observé en plantation sur des arbres de 20 mois. Conclusion. Les techniques de mycorhization contrôlée et de fertilisation avec des PN sont aptes à améliorer la croissance et la nutrition phosphatée des jujubiers en pépinières et au champ. Cependant, elles devront être testées dans d'autres sites forestiers afin d'évaluer leurs effets sur la production fourragère et fruitière des jujubiers.

Afrique occidentale / Zizyphus mauritiana / domestication / mycorhization / mycorhize à arbuscule / fertilisation / phosphate naturel / phosphore 


\section{Introduction}

Les arbres fruitiers forestiers, à usages multiples, sont exploités de manière extensive pour leurs fruits, leurs graines, le fourrage, le bois et la pharmacopée [1]. Ils participent donc à la sécurité alimentaire et nutritionnelle des populations du Sahel en leur procurant aussi des revenus non négligeables, notamment en saison sèche. L'espèce Zizyphus mauritiana Lam., communément appelée jujubier, est l'un des arbres fruitiers forestiers les plus recherchés par ces populations intéressées surtout par ses fruits. D'après une enquête faite au nord-est du Sénégal, la vente des jujubes par les villageois, en particulier par les femmes, représenterait plus de $50 \%$ des revenus tirés de la forêt par les pasteurs localisés dans la zone de forage de Mbiddi, au nord du Sénégal [2].

La recherche en matière de foresterie s'intéresse à une domestication du jujubier orientée vers l'obtention de variétés productives de fruits de qualité. La domestication est en effet une démarche itérative comprenant l'identification des plants, l'étude de leur production, la gestion de leur exploitation et la constitution d'un germplasm sélectionné [3, 4]. C'est un processus qui prend nécessairement en compte le choix, par les paysans arboriculteurs, des arbres fruitiers à domestiquer et la situation du marché de l'offre et de la demande en fruits. La domestication des arbres fruitiers forestiers proprement dite repose aussi bien sur l'amélioration de la production fruitière que sur la connaissance des contraintes du milieu susceptibles de la limiter ou même de l'inhiber.

Plusieurs approches complémentaires ont pu être envisagées pour améliorer le matériel végétal : la conservation de la ressource, la multiplication végétative pour le clonage d'individus repérés in situ pour la qualité de leur fruit, l'introduction de variétés d'arbres fruitiers domestiquées et productives, la création par hybridation de variétés améliorées qui peuvent être caractérisées du point de vue moléculaire $[1,4$, 5]. Par ailleurs, l'utilisation de certaines techniques sylvicoles adaptées à la gestion des espèces fruitières de la forêt peuvent également permettre d'améliorer la production : la taille de l'arbre, l'irrigation, la fertilisation phosphatée avec des engrais solubles ou des phosphates naturels ou la mycorhization contrôlée [6-11]. L'analyse bibliographique présentée, appuyée sur nos propres expérimentations, s'est surtout intéressée aux recherches orientées vers ces deux dernières approches. Elle a permis de mettre en évidence les avantages imputables à l'utilisation de la mycorhization contrôlée et de la fertilisation phosphatée à l'aide de phosphates naturels pour l'exploitation des jujubiers de systèmes agroforestiers à faible niveau d'intrants.

\section{Utilisations du jujubier}

Le jujubier est un petit arbre buissonnant de la famille des rhamnacées. Il mesure de $3 \mathrm{~m}$ à $8 \mathrm{~m}$ de haut. Il est très commun dans les zones sahéliennes où il se trouve soit à l'état isolé, soit, parfois, en peuplements purs. Il a une aire naturelle de répartition très large : Afrique tropicale, Amérique du Sud, Caraïbes et Asie du Sud-Est. C'est un arbre rustique à croissance lente qui pousse sur des sols très pauvres [12].

L'intérêt principal du jujubier est son fruit qui est consommé frais ou sec et qui peut servir à la préparation de galettes, de condiments et de boissons rafraîchissantes. C'est une petite drupe ovoïde allongée dont la pulpe est très riche en glucides et en vitamines A et C. Certaines variétés indiennes et thailandaises de jujubiers domestiqués produisent des fruits de la taille d'une cerise [6]. Comme la plupart des arbres, le jujubier a de nombreux usages. Les feuilles sont consommées comme légume et surtout comme fourrage d'appoint pendant la saison sèche. Son bois dense est résistant aux termites et facile à travailler pour la fabrication d'ustensiles de cuisine et d'outils. Les racines, écorces et feuilles sont utilisées dans la pharmacopée traditionnelle. L'arbre fait aussi l'objet de plantations en vergers où il fructifie 2 ans en moyenne après sa mise en place. Dans des systèmes agroforestiers, il peut être exploité en banque fourragère, haie vive ou brise-vent [13]. 


\section{Intérêts de la mycorhization contrôlée}

Les mycorhizes sont des symbioses ou associations à bénéfices réciproques entre des racines de plantes et des champignons du sol. Il existe deux principaux types de symbiose mycorhizienne : les ectomycorhizes et les mycorhizes à arbuscules.

Les mycorhizes à arbuscules concernent $90 \%$ des espèces végétales terrestres et sont universellement répandues. Elles présentent des structures typiques à l'intérieur des racines ; il s'agit de vésicules stockant des réserves et d'arbuscules, lieu d'échanges entre le champignon et la plante hôte. Les champignons responsables de ces structures appartiennent à l'ordre des glomales qui comprend trois familles : les glomacées, les acaulosporacées et les gigasporacées. Les arbuscules sont présent chez tous les glomales mais les vésicules s'observent uniquement dans les familles des glomacées et des acaulosporacées. Le champignon permetà la plante d'explorer un volume important de sol pour s'approvisionner en éléments minéraux. En retour, le champignon reçoit de la plante des photosynthétats qu'il est incapable de fabriquer lui-même. Cette symbiose améliore de manière remarquable la croissance et la nutrition phosphatée des plantes et leur permet de mieux résister à certaines maladies racinaires et d'exploiter au mieux les ressources en eau [14].

La mycorhization contrôlée est un ensemble de techniques qui consiste à isoler, cultiver, sélectionner, multiplier, incorporer et suivre le champignon dans le sol afin de produire des plants «biologiquement améliorés » par optimisation de la symbiose [15]. Si ces techniques ont commencé à être transférées dans les régions tempérées ou dans certaines régions tropicales, elles ont fait l'objet de très peu d'études en Afrique de l'Ouest.

\section{Production d'inoculum de champignons mycorhiziens à arbuscules}

Les sols ouest-africains renferment une grande diversité de champignons mycorhiziens à arbuscules qui peuvent être identifiés grâce à la morphologie de leurs spores [16-18]. Ces champignons sont inféodés aux plantes et ne présentent pas de spécificité d'hôtes.

À partir d'une collection de 13 espèces de champignons mycorhiziens à arbuscules disponibles dans notre laboratoire [16, 18], nous en avons sélectionné une, Glomus aggregatum, efficace aussi bien sur des arbres que sur des plantes annuelles $[8,11$, $19,20]$. Ce champignon a été multiplié sur des racines de mil cultivé en pot dans une chambre de culture. Après 4 mois de culture, l'inoculum obtenu se présentait sous forme de fragments de racines mycorhizées, d'hyphes et de spores à une concentration d'environ 1127 propagules viables par g de sol [8].

\section{Dépendance mycorhizienne du jujubier}

La notion de dépendance mycorhizienne exprime dans quelle mesure la symbiose est susceptible d'accroître la biomasse des plantes dans des conditions environnementales données [21]. Elle varie suivant la plante hôte, le champignon et les conditions édaphiques.

Nous avons comparé la dépendance mycorhizienne du jujubier (Z. mauritiana) à celle de douze autres arbres fruitiers forestiers indigènes ou exotiques (Adansonia digitata, Afzelia africana, Anarcadium occidentale, Aphania senegalensis, Balanites aegyptiaca, Cordyla pinnata, Dialium guineensis, Landolphia heudelottii, Tamarindus indica, Parkia biglobosa, Saba senegalensis et Sclerocaria birrea) dans un sol sableux faiblement acide ( $\mathrm{pH}$ eau $=6,4)$, pauvre en phosphore biodisponible $\left(6,6 \mu \mathrm{g} \times \mathrm{L}^{-1}\right)$ et stérilisé à l'autoclave pour éliminer la microflore native.

L'inoculation a été réalisée en comparant une souche efficace, G. aggregatum, avec une souche peu efficace, G. intraradices [8, 11]. La dépendance mycorhizienne a varié de $0 \%$ à $77 \%$. Elle a d'ailleurs été plus marquée avec G. aggregatum (figure 1). En accord avec les travaux de Habte et 


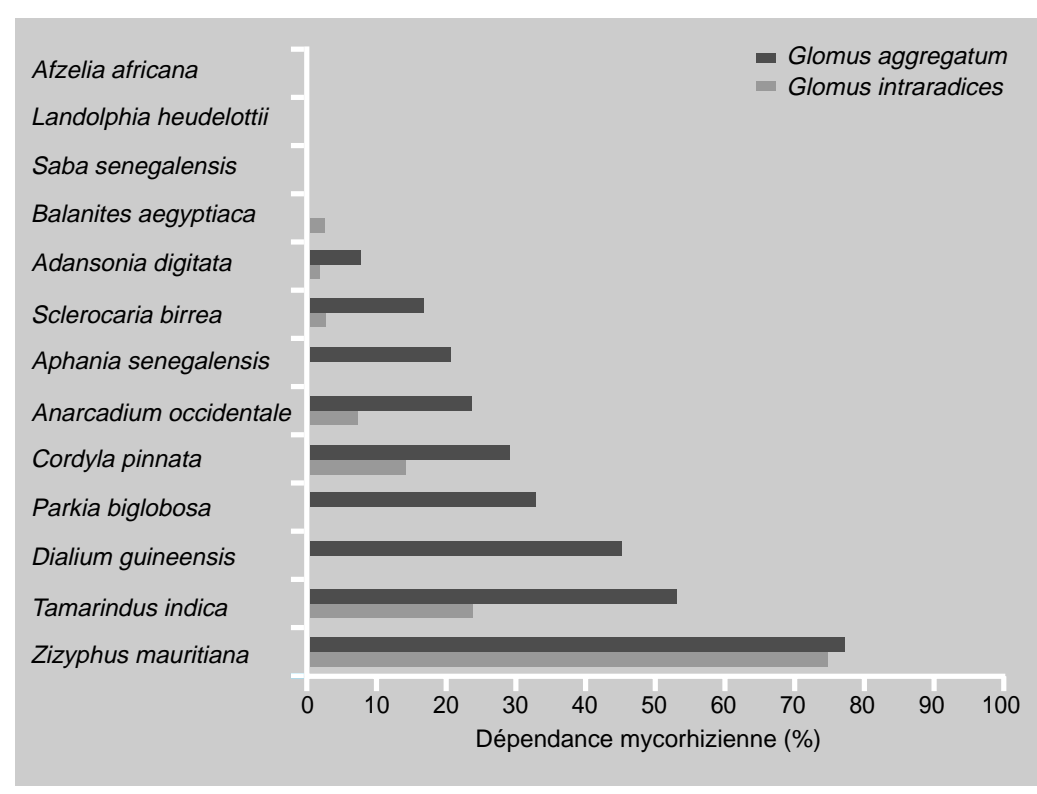

\section{Figure 1.}

Dépendance mycorhizienne de 13 arbres fruitiers forestiers vis-à-vis de deux champignons mycorhiziens à arbuscules, Glomus aggregatum et $G$. intraradices.
Manjunath [22], nous avons procédé à un classement des arbres fruitiers considérés dans notre étude en fonction de leur dépendance mycorhizienne vis-à-vis de G. aggregatum (tableau I). Pour l'espèce A. africana, qui ne présente aucune

\section{Tableau I.}

Classement de certaines espèces d'arbres fruitiers forestiers en fonction de leur dépendance mycorhizienne vis-à-vis du champignon mycorhizien à arbuscules Glomus aggregatum (Burkina Faso).

\begin{tabular}{lcc} 
Espèce fruitière & $\begin{array}{c}\text { Taux de dépendance } \\
\text { mycorhizienne (\%) }\end{array}$ & Observation \\
\hline Zizyphus mauritiana & $>75$ & Haute dépendance \\
Tamarindus indica & $>50$ & Relative bonne dépendance \\
& $<75$ & \\
$\begin{array}{l}\text { Cordyla pinnata } \\
\text { Dialium guineensis }\end{array}$ & $>25$ & Dépendance modérée \\
$\begin{array}{l}\text { Parkia biglobosa } \\
\text { Adansonia digitata }\end{array}$ & $<50$ & \\
$\begin{array}{l}\text { Anarcadium occidentale } \\
\text { Aphania senegalensis }\end{array}$ & $>0$ & Dépendance marginale \\
$\begin{array}{l}\text { Sclerocaria birrea } \\
\text { Afzelia africana } \\
\text { Balanites aegyptiaca }\end{array}$ & & \\
$\begin{array}{l}\text { Landolphia heudelottii } \\
\text { Saba senegalensis }\end{array}$ & Nulle & Aucune dépendance \\
& &
\end{tabular}

dépendance, l'inoculation a eu un effet dépressif sur la croissance. L'hypothèse explicative serait qu'une partie des photosynthétats de la plante est alors détournée à son détriment par le champignon au contact des racines [23].

Globalement, Z. mauritiana s'est révélée être l'espèce fruitière répondant le mieux à la mycorhization quel que soit le champignon inoculé (figure 1). Ces résultats permettent de conforter le choix du jujubier utilisé comme plante modèle dans l'étude des interactions entre arbres fruitiers et microorganismes intervenant dans le cycle du phosphore.

\section{Fertilisation des jujubiers mycorhizés avec des phosphates naturels}

Les sols ouest-africains sont structurellement carencés en phosphore biodisponible [24]. Cette faible disponibilité du phosphore limite considérablement la nutrition phosphatée des plantes et donc la productivité agricole et forestière. Les réserves en phosphore de ces sols ne sont plus renouvelées, car les engrais chimiques sont difficilement accessibles aux producteurs et le recyclage de la matière organique, même lorsqu'elle est disponible, ne suffit pas à remédier à cette carence. Dans ce contexte de baisse constante de la fertilité des sols, l'utilisation des phosphates naturels connaît un regain d'intérêt en Afrique de l'Ouest. Des opérations de phosphatage de fond à grande échelle ont été conduites en milieu paysan pour reconstituer les réserves en phosphore des sols agricoles [25]. Cette forme de phosphate est cependant peu disponible dans les sols à pH faiblement acide, voire neutre, très répandus en Afrique de l'Ouest [26, 27]. C'est pourquoi nous avons envisagé d'utiliser des champignons mycorhiziens à arbuscules pour mobiliser, au profit du jujubier, le phosphore de différents phosphates naturels d'Afrique de l'Ouest.

Le phosphate naturel du Burkina Faso (phosphate de Kodjari à 12,5\% de phosphore total) est réputé peu réactif, même 
dans les sols acides [26]. Nous avons montré que, dans un sol faiblement acide, une partie du phosphore libéré de ce phosphate naturel est absorbée par les jujubiers mycorhizés sans incidence sur la croissance de ces derniers $[9,28]$. On peut supposer que les jujubiers mycorhizés feraient une consommation de luxe du phosphore biodisponible.

Nous avons comparé l'efficacité du phosphate naturel du Burkina Faso à celui du Mali (phosphate de Tilemsi à $15 \%$ de phosphore total) et du Sénégal (phosphate de Taïba à 12,2 \% de phosphore total) en utilisant le triple superphosphate à $23 \%$ de phosphate soluble comme engrais de référence (tableau II). Il est apparu que, en l'absence de mycorhizes, la croissance des jujubiers était faible et cela quel qu'ait été le phosphate naturel utilisé. Le jujubier se confirme donc être hautement dépendant des mycorhizes. Lorsqu'ils sont mycorhizés, les jujubiers assimilent mieux le phosphore fourni par les différents phosphates. Cependant, le phosphate naturel du Mali a été le seul parmi les engrais naturels utilisés à avoir un effet bénéfique sur la croissance des jujubiers mycorhizés. Cet effet a alors été comparable à celui du triple superphosphate apporté à des jujubiers non inoculés. Par ailleurs, la biomasse des jujubiers mycorhizés s'est révélée fortement stimulée par le triple superphosphate, en dépit de l'effet dépressif de cet engrais sur l'intensité de la mycorhization. Les jujubiers mycorhizés ont donc une grande aptitude à utiliser les engrais phosphatés.

L'efficacité du phosphate naturel du Mali sur la croissance du jujubier a justifié que différentes doses de cet engrais soit testées vis-à-vis de la croissance de plants mycorhizés ou non, l'engrais de référence utilisé étant du triple superphosphate (tableau III).

Chez les jujubiers non mycorhizés, seuls les apports de différentes doses de triple superphosphate ont eu un effet significatif. Chez les jujubiers mycorhizés, la plus forte dose de phosphore testée $\left(125 \mathrm{~kg} \mathrm{P} \times \mathrm{ha}^{-1}\right)$ a provoqué la meilleure croissance des plants, quelle qu'ait été l'origine du phosphore appliqué. Cependant, l'assimilation du phosphore dans les tiges et les feuilles a été meilleure avec le triple superphosphate. L'intensité de mycorhization des plants a été comparable quelle que soit la dose de phosphate naturel utilisée, alors qu'elle a significativement diminué avec l'augmentation des doses de triple superphosphate. Du fait de ces résultats et pour

\section{Tableau II.}

Effets de différentes formes de phosphate sur des jujubiers inoculés ou non avec le champignon mycorhizien Glomus aggregatum, observés après 3 mois de croissance en pot (phosphate naturel (PN) à $250 \mathrm{~kg}$ phosphore $\times \mathrm{ha}^{-1}$; triple superphosphate (TSP) à $25 \mathrm{~kg}$ phosphore $\times \mathrm{ha}^{-1}$ ).

\begin{tabular}{|c|c|c|c|c|}
\hline État des jujubiers & Origine du phosphore & $\begin{array}{l}\text { Biomasse totale } \\
\text { (g) }\end{array}$ & $\begin{array}{c}\text { Phosphore des tiges } \\
\text { et feuilles }(\%)\end{array}$ & $\begin{array}{l}\text { Intensité de colonisation } \\
\qquad(\%)\end{array}$ \\
\hline \multirow[t]{5}{*}{ Non inoculé } & Sans $P$ & $0,38 \mathrm{a}$ & $0,05 \mathrm{a}$ & $0,00 \mathrm{a}$ \\
\hline & PN Mali & $0,57 \mathrm{a}$ & $0,05 \mathrm{a}$ & $0,00 \mathrm{a}$ \\
\hline & PN Sénégal & $0,29 a$ & $0,05 \mathrm{a}$ & $0,00 \mathrm{a}$ \\
\hline & PN Burkina Faso & $0,32 \mathrm{a}$ & $0,04 \mathrm{a}$ & $0,00 \mathrm{a}$ \\
\hline & TSP & $1,80 \mathrm{~d}$ & $0,20 \mathrm{e}$ & $0,00 \mathrm{a}$ \\
\hline \multirow[t]{5}{*}{ Inoculé } & Sans $P$ & $1,50 \mathrm{bc}$ & $0,14 b$ & $78,47 \mathrm{bc}$ \\
\hline & PN Mali & $1,87 \mathrm{~d}$ & $0,16 \mathrm{c}$ & $95,39 d$ \\
\hline & PN Sénégal & $1,30 \mathrm{bc}$ & $0,17 \mathrm{~cd}$ & $66,00 \mathrm{~b}$ \\
\hline & PN Burkina Faso & $1,25 \mathrm{~b}$ & 0,18 de & $87,13 \mathrm{~cd}$ \\
\hline & TSP & $2,70 \mathrm{e}$ & 0,19 e & $63,60 \mathrm{~b}$ \\
\hline
\end{tabular}




\begin{tabular}{|c|c|c|c|c|c|}
\hline État des jujubiers & $\begin{array}{c}\text { Origine } \\
\text { du phosphore }\end{array}$ & $\begin{array}{l}\text { Doses } \\
\text { de phosphore } \\
\left(\mathrm{kg} \mathrm{P} \times \mathrm{ha}^{-1}\right)\end{array}$ & $\begin{array}{l}\text { Biomasse totale } \\
\text { (g) }\end{array}$ & $\begin{array}{c}\text { Phosphore } \\
\text { des tiges et feuilles } \\
(\%)\end{array}$ & $\begin{array}{c}\text { Intensité } \\
\text { de colonisation } \\
(\%)\end{array}$ \\
\hline \multirow[t]{11}{*}{ Non inoculé } & Sans P & 0 & $0,27 \mathrm{a}$ & $0,09 a b$ & $0,00 \mathrm{e}$ \\
\hline & PN Mali & 25 & $0,21 a$ & $0,10 a b$ & $0,00 \mathrm{e}$ \\
\hline & & 50 & $0,30 a$ & $0,07 \mathrm{a}$ & $0,00 \mathrm{e}$ \\
\hline & & 75 & $0,28 a$ & $0,06 \mathrm{a}$ & $0,00 \mathrm{e}$ \\
\hline & & 100 & $0,28 \mathrm{a}$ & $0,12 \mathrm{~b}$ & $0,00 \mathrm{e}$ \\
\hline & & 125 & $0,30 \mathrm{a}$ & $0,11 a b$ & $0,00 \mathrm{e}$ \\
\hline & TSP & 25 & $1,86 \mathrm{bc}$ & 0,20 cde & $0,00 \mathrm{e}$ \\
\hline & & 50 & 2,41 cde & $0,18 \mathrm{c}$ & $0,00 \mathrm{e}$ \\
\hline & & 75 & 2,37 bcde & $0,25 \mathrm{f}$ & $0,00 \mathrm{e}$ \\
\hline & & 100 & $3,49 \mathrm{fg}$ & $0,25 f$ & $0,00 \mathrm{e}$ \\
\hline & & 125 & 2,87 ef & $0,31 \mathrm{gh}$ & $0,00 \mathrm{e}$ \\
\hline \multirow[t]{11}{*}{ Inoculé } & Sans $P$ & 0 & $1,67 \mathrm{bc}$ & 0,24 ef & $74,80 a b$ \\
\hline & PN Mali & 25 & $2,12 \mathrm{bcd}$ & 0,23 def & $64,44 \mathrm{~b}$ \\
\hline & & 50 & 2,44 cde & 0,19 cde & $67,98 \mathrm{ab}$ \\
\hline & & 75 & $2,11 \mathrm{bcd}$ & 0,19 cde & $57,38 \mathrm{bc}$ \\
\hline & & 100 & 1,82 bc & 0,23 def & 86,29 a \\
\hline & & 125 & 3,06 efg & 0,19 cde & $73,64 a b$ \\
\hline & TSP & 25 & 2,88 ef & 0,22 cdef & $57,15 \mathrm{bc}$ \\
\hline & & 50 & 2,41 cde & $0,26 \mathrm{f}$ & $76,46 a b$ \\
\hline & & 75 & 2,65 de & $0,25 f$ & $46,02 \mathrm{c}$ \\
\hline & & 100 & 2,67 de & $0,31 \mathrm{gh}$ & $48,82 \mathrm{c}$ \\
\hline & & 125 & $3,59 \mathrm{~g}$ & $0,27 \mathrm{fg}$ & $30,74 \mathrm{~d}$ \\
\hline
\end{tabular}

Dans chacune des colonnes, les moyennes suivies par la même lettre ne sont pas significativement différentes selon la méthode de Newman et Keuls au seuil de $5 \%$. Chaque valeur représente la moyenne sur douze plants.

des raisons de coût, il peut être conseillé de fertiliser les jujubiers mycorhizés en pépinière avec des phosphates naturels plutôt qu'avec du triple superphosphate, bien que ce dernier engrais ait des effets positifs avec ou sans inoculation.

\section{Mycorhization des jujubiers en pépinière et au champ}

Afin d'accélérer la croissance initiale des jeunes plants de jujubier et d'augmenter la production fourragère et fruitière de l'espèce lorsqu'elle est exploitée, il serait souhaitable de transférer la mycorhization contrôlée du jujubier dans des systèmes forestiers (vergers de fruitiers) et agroforestiers (haies vives, banques fourragères).

Dans les conditions habituelles de pépinières (substrat non stérilisé) renfermant une microflore fongique native, l'inoculation des plants par le champignon G. aggregatum a, en général, un effet bénéfique sur la biomasse totale des jujubiers (tableau IV). Cela suggérerait que la souche alors introduite serait compétitive vis-à-vis des champignons 
Tableau IV.

Effet de la mycorhization contrôlée sur des jujubiers de différentes provenances, inoculés avec Glomus aggregatum et observés après 4 mois de croissance en pépinière $(n=40)$.

\begin{tabular}{|c|c|c|c|}
\hline Provenance & Stérilisation du substrat & $\begin{array}{l}\text { Taux de colonisation } \\
\text { (\%) }\end{array}$ & $\begin{array}{l}\text { Augmentation de la croissance } \\
\text { par rapport aux plants non inoculés* }\end{array}$ \\
\hline Lery (Burkina Faso) & Stérilisé & Non déterminé & $\times 1,5$ \\
\hline Bamako (Mali) & Non stérilisé & Non déterminé & $\times 1,2$ \\
\hline KSTa (Sénégal) & Non stérilisé & 50 & $\times 1,9$ \\
\hline Gola (Thaïlande) & Non stérilisé & 55 & $\times 3$ \\
\hline
\end{tabular}

mycorhiziens à arbuscules natifs des substrats de pépinières. Par ailleurs, il apparait également que la réponse des jujubiers à l'inoculation différerait selon la provenance des jujubiers. Ce sont ceux de Gola (Thailande) qui répondraient le mieux à l'inoculation, alors que ceux de Bamako (Mali) y seraient le moins sensibles. Ainsi, des jujubiers provenant de Gola, transplantés dans la station de recherche de l'Institut sénégalais de Recherches agricoles (Isra) à Bandia (sud du Bassin arachidier, Sénégal), ont disparu au bout de 5 mois lorsque les plants n'avaient pas été inoculés, alors que, pour les plants inoculés, le taux de survie était de $80 \%$ (comm. pers.).

Une expérimentation destinée à évaluer l'influence d'une inoculation avec G. aggregatum sur la croissance du jujubier en plantation a été réalisée dans le cadre d'une collaboration entre l'Isra, la Direction des Eaux et forêts, de la chasse et de la conservation des sols (DEFCCS), l'Institut de Recherche pour le développement (IRD) et le Projet de Reboisement villageois dans le nordouest du Bassin arachidier (PREVINOBA). Cet essai de mycorhization contrôlée de l'espèce a été mis en place dans un champ de la localité de Colobane Thiombane (Département de Tivaouane, Sénégal). Après 4 mois de croissance en pépinière, il a été constaté que l'inoculation avait stimulé la biomasse totale du jujubier d'un facteur multiplicateur de 1,9 (tableau IV). Après 20 mois en plantation, les jujubiers inoculés présentaient une hauteur et un diamètre au collet significativement améliorés par rapport aux plants non inoculés (tableau V).

\section{Conclusion}

La mycorhization contrôlée et la fertilisation phosphatée avec des phosphates naturels sont des techniques efficaces pour stimuler la croissance et la nutrition phosphatée des jujubiers en pépinière et au champ. Malgré les résultats encourageants obtenus au champ, ces techniques devront être éprouvées dans d'autres sites forestiers afin d'évaluer leurs effets sur la production fruitière et fourragère des jujubiers. Elles pourront ensuite être prises en compte dans la stratégie de domestication de Z. mauritania.

Les recherches effectuées sur l'efficacité de la mycorhization et de l'utilisation des phosphates naturels gagneront à être

\section{Tableau V.}

Effet de la mycorhization contrôlée sur des jujubiers (provenant de Keur Serigne Touba, Sénégal) inoculés avec Glomus aggregatum, observés 20 mois après leur mise en place en plantation $(n=60)$.

\begin{tabular}{lcc} 
État des jujubiers & $\begin{array}{c}\text { Hauteur de la tige principale } \\
(\mathrm{m})\end{array}$ & $\begin{array}{c}\text { Diamètre au collet } \\
(\mathrm{cm})\end{array}$ \\
\hline Inoculé & $1,55 \mathrm{a}$ & $2,8 \mathrm{a}$ \\
Non inoculé & $1,04 \mathrm{~b}$ & $1,5 \mathrm{~b}$
\end{tabular}

Dans chacune des colonnes, les moyennes suivies par la même lettre ne sont pas significativement différentes selon la méthode de Newman et Keuls au seuil de $5 \%$. 
complétées par la mise en œuvre de méthodes d'amélioration du matériel végétal et de multiplication végétative horticole et in vitro. Conjointement, l'introduction de variétés productives provenant d'Asie du Sud-Est devra être entreprise [2].

Un autre volet d'étude touchant la domestication du jujubier est lié à sa grande sensibilité aux nématodes phytoparasites Meloidogyne javanica qui pourrait en faire un réservoir du pathogène [2]. Cette sensibilité aux nématodes à galles pose des problèmes pour l'utilisation de cet arbre fruitier dans les systèmes agroforestiers. Le rôle antagoniste de champignons mycorhiziens à arbuscules sur M. javanica déjà mis en évidence par ailleurs [29] devra être vérifié dans le cas de la culture du jujubier.

\section{Références}

[1] Okafor J.C., Amélioration des essences forestières donnant des produits comestibles, Unasylva 165 (1991) 17-23.

[2] Anonyme, Projet ligneux fruitiers (Sénégal), in : Isra/CRDI (Éd.), Rapport final d'exécution scientifique et technique (1991-1997), Direction des Productions forestières (DRPF), Isra/CRDI, Sénégal, 1997, 83 p.

[3] Leakey R.R.B., Simons A.J., The domestication and commercialization of indigenous trees in agroforestry for the alleviation of poverty, Agroforest. Syst. 38 (1998)165-176.

[4] Leakey R.R.B., Tomich T.P., Domestication of tropical trees: from biology to economics and policy, in : Buck L.E., Lassoie J.P., Fernandes E.C.M. (Éd.), Agroforestry in sustainable agriculture systems, New York, USA, 1999, pp. 319-338.

[5] Leakey R.R.B., Potential for novel food products from agroforestry trees: a review, Food Chem. 66 (1999) 1-14.

[6] Bajwa G.S., Sandhu H.S., Bal J.S., Effect of pruning severety on growth and bearing of ber, Indian J. Hortic. 22 (1988) 203-206.

[7] Bâ A.M., Duponnois R., Danthu P., Diallo I., Guissou T., Sanon K.B., Sacko O., Plenchette C., Utilisation des phosphates naturels par des jujubiers mycorhizés, in : ISSS (Éd.), Résumé du $16^{\mathrm{e}}$ congrès mondial de Sciences du sol, 20-26 août, Montpellier, France, 1998, p. 21.
[8] Guissou T., Bâ A.M., Ouadba J.M., Guinko S., Duponnois R., Responses of Parkia biglobosa (Jacq.) Benth., Tamarindus indica L. and Zizyphus mauritiana Lam. to arbuscular mycorrhizal fungi in a phosphorus deficient soil, Biol. Fert. Soils 26 (1998) 194-198.

[9] Guissou T., Bâ A.M., Guinko S., Duponnois R., Plenchette C., Influence des phosphates naturels et des mycorhizes à vésicules et à arbuscules sur la croissance et la nutrition minérale de Zizyphus mauritiana Lam. dans un sol à pH alcalin, Ann. Sci. For. 55 (1998) 925-931.

[10] Bâ A.M., Guissou T., Duponnois R., Danthu P., Konde S., Baba V., Sacko O., Sidibé D., Plenchette C., Controlled mycorrhization of jujubes tree (Zizyphus mauritiana Lam.) to increase the productivity of plantations in West Africa, in: TSBF (Ed.), Opportunities and chalenges for African agriculture, Nairobi, Kenya, 1999, p. 21.

[11] Bâ A.M., Plenchette C., Danthu P., Duponnois R., Guissou T., Functional compatibility of arbuscular mycorrhizae with thirteen tropical fruit trees in Senegal, Agroforest. Syst. 95 (2000) 95-105.

[12] Depommier D., Ziziphus mauritiana Lam., culture et utilisation en pays Kapsiki (NordCameroun), Bois For. Trop. 218 (1988) 57-63.

[13] Bonkoungou E.G., Djimde M., Ayuk E.T., Zoungrana I., Tchoundjeu Z., Taking stock of agroforestry in the sahel-harvesting results for the future, in : Icraf (Ed.), End of phase report 1989-1996, Nairobi, Kenya, 1998, pp.1-58.

[14] Smith S.E., Read D.J., Mycorrhizal symbiosis, Academic Press, second edition, Oxford, UK, 1997.

[15] Garbaye J., Utilisation des mycorhizes en sylviculture, in : Lavoisier (Éd.), Les mycorhizes des arbres et plantes cultivées, Paris, France, 1991, pp. 197-248.

[16] Bâ A.M., Dalpé Y., Guissou T., Les glomales d'Acacia holosericea A. Cunn. ex G. Don. et d'Acacia mangium Willd. : diversité et abondance relative des champignons mycorhiziens à arbuscules dans deux types de sols de la zone nord et sud soudanienne du Burkina Faso, Bois For. Trop. 250 (1996) 5-18.

[17] Diallo A.T., Samb P.I., Ducousso M., Arbuscular mycorrhizal fungi in the semi-arid areas of Senegal, Euro. J. Soil Biol. 35 (2000) 65-75.

[18] Dalpé Y., Diop T., Plenchette C., Gueye M., Biodiversity of glomales with soil depth under Faidherbia albida in Senegal, Mycorrhiza (2001), sous presse. 
[19] Bâ A.M., Guissou T., Rock phosphate and mycorrhizas effects on growth and nutrient uptake of Faidherbia albida (Del.) in an alkaline sandy soil, Agroforest. Syst. 34 (1996) 129-137.

[20] Dianou D., BâA.M., Réponse de Vigna unguiculata (L.) Walp. à l'inoculation de rhizobactéries et de champignon mycorhizien en présence de phosphate naturel, Ann. Univ. Ouagadougou série B Vol. VII (1999) 107-122.

[21] Plenchette C., Fortin J.A., Furlan V., Growth responses of several plant species to mycorrhizae in a soil of moderate P-fertility, I : mycorrhizal dependency under field conditions, Plant Soil 70 (1983) 199-209.

[22] Habte M., Manjunath A., Categories of vesicular-arbuscular mycorrhizal dependency of host species, Mycorrhiza 1 (1991) 3-12.

[23] Plenchette C., Utilisation des mycorhizes en agriculture et horticulture, in : Lavoisier (Éd.), Les mycorhizes des arbres et plantes cultivées, Paris, France, 1991, 131-179.

[24] Piéri C., Fertilité des terres de savanes. Bilan de trente années de recherche et de développement au Sud du Sahara, Ministère de la Coopération et Cirad, Paris, France, 1989.
[25] Snrech S., Les phosphates rocheux au secours de l'agriculture africaine ? Club du Sahel, Bull. Info. 15 (1996) 26-29.

[26] Truong B., Pichot J., Beunard P., Caractérisation et comparaison des phosphates naturels tricalciques d'Afrique de l'Ouest en vue de leur utilisation directe en agriculture, Agron. Trop. 33 (1977) 136-145.

[27] Bâ A.M., Bazié M., Guissou T., Effet du phosphate naturel de Kodjari sur la croissance et la nutrition minérale de Faidherbia albida Del. en présence ou non de mycorhizes à vésicules et à arbuscules, in : Cirad-Forêt (Éd.), Les parcs à Faidherbia, Cah. Scientif. 12 (1996) 237-244.

[28] Guissou T., Bâ A.M., Guinko S., Plenchette C., Duponnois R., Mobilisation des phosphates naturels de Kodjari par des jujubiers (Zizyphus mauritiana Lam.) mycorhizés dans un sol acidifié avec de la tourbe, Fruits 55 (2000) 187-194.

[29] Duponnois R., Cadet P., Interactions of Meloidogyne javanica and Glomus sp. on growth and nitrogen fixation of Acacia, AfroAsian J. Nematol. 4 (1994) 228-233.

\section{Micorrización controlada y fertilización fosfatada: aplicaciones para la domesticación del azufaifo africano.}

Resumen - Introducción. El azufaifo, Zizyphus mauritiana Lam., es un árbol frutal forestal muy buscado por sus frutos por las poblaciones del Sahel. Se ha emprendido la domesticación de esta especie mediante técnicas silvícolas como la micorrización controlada y la fertilización fosfatada con fosfatos naturales (FN). El documento presenta una síntesis de los resultados obtenidos sobre estos temas. Material y métodos. Se ha estudiado la dependencia micorrízica del azufaifo y otras doce especies de árboles frutales forestales respecto de dos hongos micorrízicos arbusculares Glomus aggregatum y G. intraradices. Además, se ha evaluado la capacidad del árbol para movilizar fósforo a partir de FN del África occidental en un suelo arenoso, pobre en fósforo asimilable y con $\mathrm{pH}$ débilmente ácido. Posteriormente, se llevaron en campo los azufaifos inoculados con G. aggregatum para observar sus parámetros de crecimiento. Resultados y discusión. Entre las trece especies de árboles frutales estudiadas, el azufaifo se mostró la más dependiente de la simbiosis micorrízica con G. aggregatum que actúa sobre su crecimiento y su alimentación en fósforo asimilable. Los azufaifos micorrizados movilizan más eficazmente el fósforo de los FN de Tilemsi (Malí) que el de los FN de Taiba (Senegal) y Kodjari (Burkina Faso). En viveros, la micomización controlada tiene efectos benéficos sobre el crecimiento de azufaifos de diversas procedencias. Este efecto benéfico de la inoculación fue también observado en plantación en árboles de 20 meses. Conclusión. Las técnicas de micorrización controlada y de fertilización con FN tienen la capacidad de mejorar el crecimiento y la nutrición fosfatada de azufaifos en viveros y en campo. Sin embargo, deberán probarse en otros sitios forestales para evaluar sus efectos sobre la producción forrajera y frutal de los azufaifos.

África occidental / Zízyphus mauritiana / domesticación / infección de micorrizas / micorrizas arbusculares / aplicación de abonos / fosfato mineral / fosforo 